\title{
Effect of buccal corridors on smile esthetics
}

\author{
Ramya KS ${ }^{1 *}$, Mahesh $\mathbf{H V}^{2}$ \\ ${ }^{\mathbf{1}}$ Assistant Professor, ${ }^{2}$ Associate Professor, ${ }^{1,2}$ Dept. of Dentistry, ${ }^{1}$ Mysore Medical College \& Research Institute, Karnataka, ${ }^{2}$ Mandya \\ Medical College \& Research Institute, Mandya, Karnataka, India \\ *Corresponding Author: Ramya KS \\ Email: orthoramya@gmail.com
}

\begin{abstract}
Objective: To evaluate the influence of buccal corridor space on smile attractiveness as judged by lay persons and orthodontists. Materials and Methods: Colour photographs depicting entire face of 10 aesthetically pleasing subjects (5 females and 5 males) were taken. Digital alteration of maxillary posterior dentition area was done in all photographs to get a range of smile fullness i.e. Narrow, medium and broad. The digitally modified images were presented to a panel of ten lay persons and ten orthodontists for image comparison and determining smile attractiveness.

Results: The difference in the judgement of lay persons and orthodontists pertaining to the influence of buccal corridors on smile aesthetics was not significant. Both lay persons and orthodontists rated small buccal corridor space as having more attractive smile than the ones with large buccal corridor space.

Conclusion: Minimal buccal corridors can be called as one of the preferred aesthetic feature and large buccal corridor spaces could be considered for orthodontic corrections.
\end{abstract}

Keywords: Buccal corridor space, Smile aesthetics \& Orthodontists.

\section{Introduction}

Face is one of the key features of Physical attractiveness of an individual. Studies have reported face as being the most important component of aesthetic perception of any person. ${ }^{1}$ Mouth and eyes are further important components for determining aesthetic value in the face. ${ }^{2}$ Since aesthetic consideration is one of the foremost reasons for patients to consider orthodontic treatment, it becomes the job of orthodontist to properly evaluate and understand the factors influencing aesthetics of any person. ${ }^{3}$

The current generation aesthetic values differ from those that were standard a few decades ago. Aesthetic perception is also a variable factor and depends on individual and is greatly influenced by their personal experience and social environment, this is one of the reasons why professional opinion regarding evaluation of facial aesthetics may not always coincide with the perception and expectation of patients or lay people.

The smile aesthetics is one of the most important contributors to the facial aesthetics. Further the factors contributing to smile aesthetics include the area of gingival display; colour, contour, texture and height of the gingiva; the presence of smile arc; the teeth by its contributing factors of size, shape, shade and alignment and the buccal corridor space. ${ }^{4-7}$

How much the buccal corridors influence the smile attractiveness is a subject of controversy. The paradigm shift from occlusion to aesthetics emphasises the need to explore the variables affecting smile. Frush and Fisher were the first to introduce the concept of buccal corridor space and its influence on smile aesthetics. Frush \& Fisher in the year 1958 termed the space between the facial surface of the posterior teeth and corner of lips when the person smiles as 'buccal corridors'. The other terminologies for buccal corridor space are negative spaces or black spaces. They felt that in a patient wearing denture the absence of buccal corridor space gives the patient an unnatural "Denture appearance". 8

The literature in prosthodontics broadly describes any smile which is lacking in buccal corridors to be denture like and unrealistic and the orthodontists appear to prefer to have minimal buccal corridor space for a more appealing smile. Literature review presents with conflicting data pertaining to the influence of buccal corridor space on smile aesthetics. Hence, this study was designed with the purpose to evaluate the influence of buccal corridor space on smile attractiveness when judged by lay persons and orthodontists.

\section{Materials and Methods}

Smiling, full face colour photographs of ten aesthetically pleasing subjects ( 5 males \& 5 females) were taken. Only those subjects who had good alignment of teeth and who had all teeth up to second molars were chosen for the study.

The frontal view photographs of the subjects were taken and as slight variations in patient to film distances could be expected which could result in slight inappropriateness in measuring the exact linear buccal corridor width. Hence, to overcome the errors the buccal corridor widths and smile fullness were expressed as percentage of the commissure width. As the dentition can fill the smile maximum only up to the innermost aspect of the commissure, smile fullness and buccal corridor width were expressed as ratios of inner commissure width.

The visible maxillary dentition width was divided by the inner commissure width to calculate the smile fullness, the difference between the visible maxillary dentition width and the inner commissure width divided by the inner commissure width (Fig. 1) was taken as buccal corridor. Both the ratios were expressed as percentages. The sum of the two percentages for a given image would equal $100 \%$. 
To make modified images of varying sizes of buccal corridor space, each photograph was first copied and imported to adobe photoshop 7.0. All the images were set to the same magnification. Three modified photographic images were produced for each of the ten subjects to have a range of smile fullness i.e. narrow, medium and broad. To obtain the modified images, the original image was first altered to produce a broad visible dentition with filled buccal corridors. The intercanine width was unaltered to give a realistic appearance. Then the altered image was further reduced to produce two subsequent images with narrower dentitions (medium and large buccal corridor space) (Fig. 2).

The only difference in the altered images and the original image was the amount of buccal corridor space (or smile fullness). These images were presented to judges i.e. lay persons and ten orthodontists. The judges were instructed to choose the smile they preferred from each of the three images for each subject and the data was recorded.

\section{Results}

The results showed consistent relationship between the amount of smile fullness (buccal corridor space) and smile attractiveness in the study. As graphs 1 and 2 depict, laypersons chose smiles with small buccal corridor space in $41 \%$ of the sample images provided and orthodontists chose smiles with small buccal corridor space in $54 \%$ of the sample images provided. Thus, it can be clearly said that smaller the buccal corridor space (broader the smile fullness) the more attractive the smile as per both the groups. Similarly, larger the buccal corridor space (narrow smile fullness), less attractive the smile as per both the groups.

On an average broad smile fullness (2\% buccal corridor space) was rated the best, by both orthodontists and laypersons. Followed by medium smile fullness (15\% buccal corridor space) and narrow smile fullness (22\% buccal corridor space).

This indicates that both the laypersons and orthodontists prefer smiles that are visibly filled with the teeth between both the commissures. The Smiles in subjects who had large buccal corridor space were considered as less attractive by both the groups.
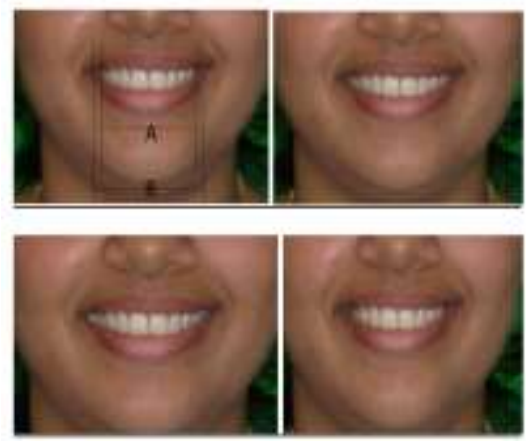

Fig. 1

Graph 1: Smile attractiveness related to buccal corridor width as judged by laypersons.

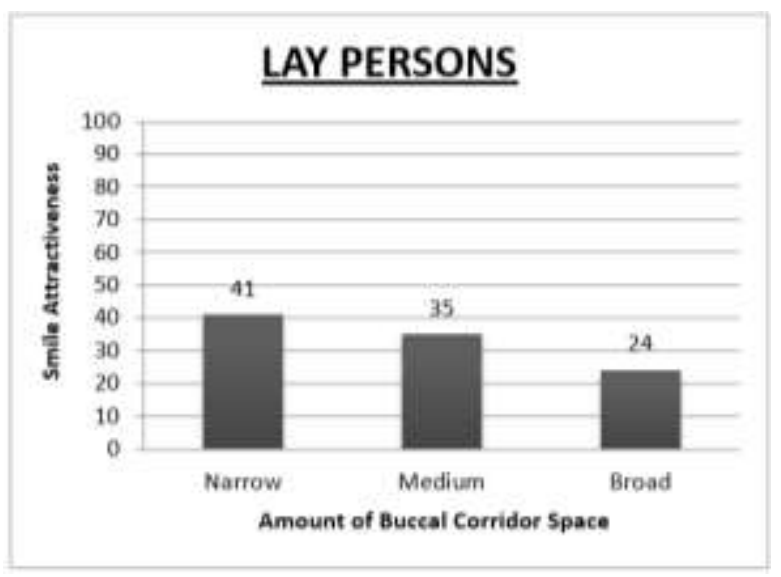

Graph 2: Smile attractiveness related to buccal corridor width as judged by orthodontists.

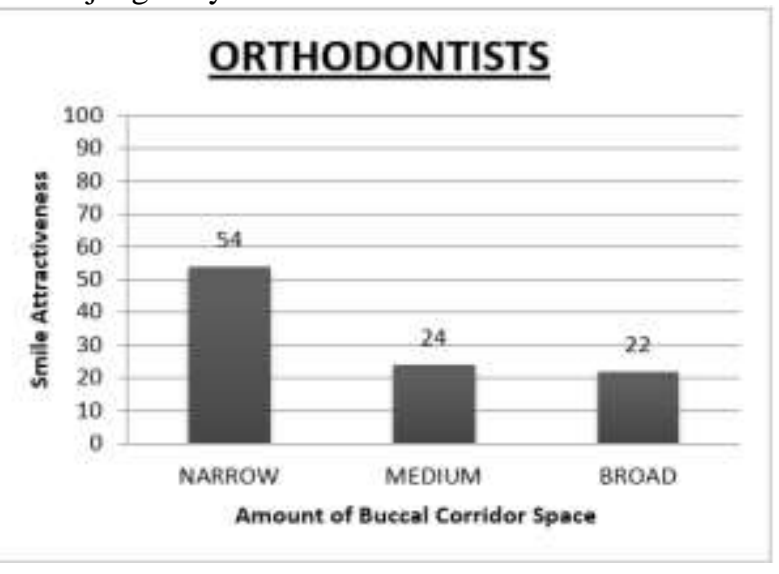

\section{Discussion}

Smile aesthetics when analysed using full facial view differs from smile analysed using dental view. Studies have stated that when the entire face is taken in context the buccal corridor space influences the smile attractiveness of a person. ${ }^{3,9}$ This study evaluated smile utilizing frontal full facial view photographs.

As Margaret Wolfe Hungerford famously said "Beauty is in the eye of beholder", aesthetic perception is subjective. There have been many studies that have evaluated only persons' perception of buccal corridors. ${ }^{9-12}$ This study was designed with the intention to determine whether laypersons and orthodontists have similar or contravening opinion regarding the influence of buccal corridors on smile aesthetics. The results however revealed that both orthodontists and laypersons preferred smiles with minimal or no buccal corridors. These findings however are in sharp contradiction with a study by Hulsey who reported that laypersons had no preference regarding buccal corridor width, and width variation seemed to be of no significance in determining smile attractiveness. ${ }^{10}$ The reason for this result by Hulsey might be due to usage of used limited to mouth. As there are multiple factors like facial makeover, skin tone differences, lip stick applications which have considerable influences on aesthetics of smile. In this study picture of full face was taken and the findings of this study 
parallel a trend noted by many authors who opine that that small buccal corridors are more attractive ${ }^{9,13-15}$

The results of this study indicates that less buccal corridor space is perceived to result in better smile aesthetics as judged by both the groups which can be taken from the orthodontists point of view that minimizing buccal corridors will improve smile aesthetics. Some of the treatment procedures that can be considered for reducing the buccal corridor space are such as maxillary arch expansion and increasing the torque in posterior teeth. However, in a normal maxilla reduction of buccal corridor space should not be considered a rationale for the purpose of maxillary expansion. Increasing the crown torque in the posterior segment to minimize negative spaces is also not substantiated. ${ }^{16}$ Also it should be finally noted that smile fullness (buccal corridor space) is just one feature apart from various features that affect smile attractiveness.

\section{Conclusion}

Less buccal corridors was judged to be more attracive by both laypersons and orthodontists than large buccal corridors. There was no significant difference between judgement of laypersons and orthodontists in determining the smile attractiveness. Having minimal buccal corridor space can be called as one of the preferred aesthetic feature in both men and women and those individuals with large buccal corridor space can be considered as subjects for orthodontic treatment from aesthetic point of view.

\section{Source of Funding}

None.

\section{Conflict of Interest}

None.

\section{References}

1. Peck S, Peck L. Selected aspects of the art and science of facial esthetics. Semin Orthod. 1995;1(2):105-26.
2. Shaw WC, Rees G, Charles CR. The influence of dentofacial appearance on the social attractiveness of young adults. Am J Orthod. 1985;87(1):21-6.

3. Flores-Mir C, Silva E, Barriga MI, Lagravere MO, Major PW. Lay person's perception of smile aesthetics in dental and facial views. J Orthod. 2004;31(3):204-09.

4. Kokich VO, Kayak HA, Shapiro PA. Comparing the perception of dentists and lay people to altered dental esthetics. J Esthetic Dent. 1999;11(6):311-24.

5. Sabri R. The eight components of a balanced smile. J Clin Orthod. 2005;39(3):155-67.

6. Morley J, Eubank J. Macroesthetic elements of smile design. $J$ Am Dent Assoc. 2001;132(1):39-45.

7. Sarver DM, Ackerman MB. Dynamic smile visualization and quantification: Part 2.Smile analysis and treatment strategies. Am J Orthod Dentofac Orthop. 2003;124(2):116-27.

8. Frush JP, Fisher RD. The dynesthetic interpretation of the dentogenic concept. J Prosthetic Dent. 1958;8:581.

9. Moore T, Southard RA, Casko JS, Qian F, Southard TE Buccal corridors and Smile esthetics. Am J Orthod Dentofacial Orthop 2005;125:208-13.

10. Hulsey TM. An esthetic evaluation of lip-teeth relationships present in the smile. Am J Orthod. 1970;57(2):132-44.

11. Johnson DR and Smith RJ. Smile esthetics after orthodontic treatment with and without extraction of four first premolars. Am J Orthod Dentofacial Orthop. 1995;108(2):162-7.

12. Kim E. Gianelly AA. Extraction vs non-extraction widths and smile esthetics. Angle Orthod. 2003;73(4):354-8.

13. Parekh SM, Fields HW, Rosensteil SF, Beck FM. Attractiveness of variations in smile arc and buccal corridor spaces as judged by orthodontists and laymen. Angle Orthod. 2006;76(4):557-63.

14. Martin AJ, Buschang PH, Boley JC, Taylor RW, Mc Kinney TW. The impact of buccal corridors on smile attractiveness. Eur J Orthod. 2007;29(5):530-7.

15. Ritter DE, Gandhi LG, Pinto Ados S, locks A. Esthetic influence of buccal corridor during smiling. Angle Orthod. 2006;76(2):198-203.

16. Ker AJ, Chan R, Beck M, Rosensteil S. Esthetics and smile characteristics from laypersons perspective: A computer based survey study. J Am Dent Assoc. 2008;139(10):1318-27.

How to cite: Ramya KS, Mahesh HV. Effect of Buccal corridors on smile esthetics, Indian $J$ Orthod Dentofacial Res. 2020;6(1):9-11. 